American Journal of Pharmacology and Toxicology 4 (4): 174-176, 2009

ISSN 1557-4962

(C) 2009 Science Publications

\title{
The "Forgotten Side Effect" of Thiazides
}

\author{
Arnon Blum \\ Department of Internal Medicine, Baruch Padeh Poria Medical Center, \\ Lower Galilee 15208, Israel
}

\begin{abstract}
Problem statement: Thiazide diuretics are derived from benzodiazines. They inhibit $\mathrm{Na}^{+} / \mathrm{Cl}^{-}$re-absorption from the distal convoluted tubules in the kidneys by blocking the thiazidesensitive $\mathrm{Na}^{+} / \mathrm{Cl}^{-}$symporter. They are recommended as first line treatment for hypertension in the US $(\text { JNC VII })^{[1]}$ guidelines and in the National Institute of Health and Clinical Excellence/British Hypertension Society guidelines ${ }^{[2]}$ and in the European $(\mathrm{ESC} / \mathrm{ESH})^{[3]}$ guidelines. They have been shown to prevent hypertension-related morbidity and mortality, although the long term mechanism is not fully understood. Approach: Side effects include hypokalemia, increased serum cholesterol, impaired glucose intolerance, diabetes mellitus and impotence. Long term usage of thiazides is also linked to increased levels of homocysteine and a paradoxical effect in Diabetes Insipidus, where they reduce urine volume. However, there is a side effect that was ignored during the last 30 years-and that is the association with gall bladder disease. We would like to stress this side effect. Results: Thiazide diuretics have a "forgotten" side effect that was not mentioned among the well-known side effects of the drug. Conclusion: The mechanism is not completely understood, but the association is clear and physicians should be aware of that side effect.
\end{abstract}

Key words: Side effects, acute cholecystitis, thiazide diuretics

\section{INTRODUCTION}

Thiazides and gall bladder disease: According to a recent publication in the American Family Physician $12 \%$ of the population in the USA are affected by Gallstone disease. Several factors are associated with an increased occurrence of gallstone formation that include body habitus (obesity (BMI greater than $30 \mathrm{~kg} \mathrm{~m}^{-2}$ ), rapid weight loss, cyclic weight loss), childbearing, drugs (ceftriaxone (Rocephin), postmenopausal estrogens, total parenteral nutrition), ethnicity (native Americans (Pima Indian), Scandinavian), female gender, heredity (first degree relatives), ileal disease, ileal resection or bypass and an increasing age ${ }^{[4]}$. However, 30 years ago Rosenberg et al. ${ }^{[5]}$ have found an association between acute cholecystitis and the use of thiazide diuretics. An evaluation of 419 patients with acute cholecystitis and 1676 control patients yielded a relative risk estimate of 2.0 for subjects who used thiazides in the month before admission, as compared with subjects who had never used these drugs. There was a significant trend of increasing relative risk with increasing duration of use and the estimate for subjects who had used thiazides for 5 or more years was 2.9. The association was not explained by confounding due to the indications for thiazide use, such as hypertension or other factors (obesity or the use of other drugs) ${ }^{[5]}$. A
Swedish study that was performed from 1974-1983 found that 91 patients (47 women and 44 men) that were admitted with acute cholecystitis had purchased thiazides in the last year, compared with a control group of 364 people. The relative risk for recent purchase and the development of acute cholecystitis was 2:1. No significant association was found between past purchase and acute cholecystitis. Only thiazides were purchased in a higher proportion of patients with acute cholecystitis than controls. The rapid return of risk to normal when treatment with thiazides was stopped suggests that thiazides do not induce gall stones but cause cholecystitis ${ }^{[6]}$. A case control study that was conducted in the US among women who had cholecystectomy found that the risk of gallstone surgery among women who had used thiazides relative to those who had not used this drug was 2.2. Standardization of age, race, high blood pressure and pregnancy did not alter the estimate of relative risk of 1.9. When the analysis was limited to the 120 cases who had matched controls, the relative risk was 2.7. The effect of thiazide use on gallstone disease appeared to be modified only by overweight. Among women who were not overweight, the relative risk was 5.0, whereas it was 1.0 among women who were overweight. No increase in risk was found with increasing duration of use of thiazides ${ }^{[7]}$. 
A prospective study that examined the association between thiazide use and cholecystectomy (a surrogate for symptomatic cholelithiasis) in a cohort of 81,351 US women aged 30-55 years between the years 1980 and 2000, found that there was a modest positive relation between the use of thiazide diuretics and cholecystectomy and the multivariate relative risk for current users was 1.39. These results suggested a specific thiazide effect since no association was found between furosemide use and cholecystectomy, or by concomitant use of other antihypertensive agents like beta blockers, calcium channel blockers and ACE inhibitors ${ }^{[8]}$.

\section{What is the mechanism:}

Animal studies: Animal studies have shown that thiazides affect the contraction of smooth muscle ${ }^{[9]}$. The spasmogenic effect of histamine, acetylcholine and barium chloride on the isolated muscle of rabbits is inhibited by thiazides ${ }^{[10]}$. It is possible that thiazides interfere with the contractile activity of the gallbladder smooth muscle wall and may impair its rate of emptying, leading to bile stasis and stone formation.

Human studies: A pilot study that was conducted with 10 healthy volunteers ( 7 males, 3 females, mean age of 31 years) that were administered hydrochlorthiazide for 3 weeks (25 mg daily) found that cholesterol concentration was increased in 7 of the subjects, from a mean of $69 \%$ before to $81 \%$ during the treatment (an increase of $17 \%$ ). So this human study suggests that treatment with thiazide preparations may influence biliary lipid composition ${ }^{[11]}$. Other possible mechanisms include the abnormalities of lipoprotein metabolism (increase in triglyceride levels) during treatment with thiazides ${ }^{[12]}$. Since hypertriglyceridemia is linked to enhanced biliary secretion of cholesterol, with a subsequent increase in the cholesterol concentration of bile, it is tempting to speculate that acute cholecystitis can occur during thiazide therapy ${ }^{[12]}$. Thiazides also induce glucose intolerance, leading to gallbladder hypomotility, a condition that is associated with the development of both cholesterol and pigment gallstones, as well as with acute acalculous cholecystitis $^{[13,14]}$.

Because thiazides frequently cause hypokalemia and hypomagnesemia, an adverse effect on the gallbladder could be due in part to deficient levels of potassium and magnesium, 2 dietary factors that were found to have inverse relationships with gallstones and cholecystectomy ${ }^{[8]}$.

\section{CONCLUSION}

It seems that thiazide diuretics have a "forgotten" side effect that was not mentioned among the wellknown side effects of the drug. The mechanism is not completely understood, but the association is clear and physicians should be aware of that side effect.

\section{REFERENCES}

1. Chobanian, A.V., G.L. Bakris and H.R. Black et al., 2003. The seventh report of the joint national committee on prevention, detection and treatment of high blood pressure. J. Am. Med. Assoc., 289: 2560-2572. PMID: 12748199

2. National Institute for health and Clinical Excellence, 2006. Hypertension: Management of hypertension in adults in primary care. http://www.nice.org.uk/CG034

3. European Society of Cardiology, 2009. CVD prevention in clinical practice. http://www.escardio.org/knowledge/guidelines/CV

4. Bellows, C., D.H. Berger and R.A. Crass, 2005. Management of gallstones. Am. Fam. Phys., 72: 637-642. PMID: 16127953

5. Rosenberg, L., S. Shapiro, D. Slone, D.W. Kaufman, O.S. Miettinen and P.D. Stonlley, 1980. Thiazides and acute cholecystitis. N. Eng. J. Med., 303: 546-548. PMID: 7402220

6. Van der Linden, W., B. Ritter and G. Edlund, 1984. Acute cholecystitis and thiazides. Br. Med. J., 289: 654-655. PMID: 6434025

7. Kakar, F., N.S. Weiss and S.A. Strite, 1986. Thiazide use and the risk of cholecystectomy in women. Am. J. Epidemiol., 124: 428-433. PMID: 3740043

8. Leitzman, M.F., C.J. Tsai, M.J. Stampfer, W.C. Willet, E. Giovannucci, 2005. Thiazide diuretics and the risk of gallbladder disease requiring surgery in women. Arch. Int. Med., 165: 567-573.

9. Davila, D. and T. Davila, 1981. Thiazide diuretics inhibit contractions of isolated smooth muscles. Pharmacology, 22: 108-112. PMID: 7208592

10. Fries, E.D., A. Wanko and H.W. Schnaper et al., 1960. Mechanism of the altered blood pressure responsiveness produced by chlorthiazide. J. Clin. Invest., 39: 1277-1281.

11. Angelin, B., 1989. Effect of Thiazide treatment on biliary lipid composition in healthy volunteers. Eur. J. Clin. Pharmacol., 37: 95-96. PMID: 2591472 
12. Ames, R.P. and P. Hill, 1976. Evaluation of serum lipid levels during diuretic therapy of hypertension. Am. J. Med., 61: 748-757. PMID: 984073

13. Einarsson, K. and B. Angelin, 1986. Hyperlipoproteinemia, hypolipidemic treatment and gallstone disease. Atheroscler Rev., 15: 67-97.
14. Thompson, W.G., 1990. An assault on old friends: Thiazide diuretics under siege. Am. J. Med. Sci., 300: 152-158. PMID: 2240006 